Short communication

\title{
An additional primary proteolytic processing site in merozoite surface protein-1 of Plasmodium berghei
}

\author{
Mark F. Wiser*, Carole S. Toebe ${ }^{1}$, Gregory J. Jennings ${ }^{2}$ \\ Department of Tropical Medicine, Tulane University School of Public Health and Tropical Medicine, 1501 Canal Street, \\ New Orleans, LA 70112, USA
}

Received 10 September 1996; revised 26 November 1996; accepted 29 November 1996

Keywords: Plasmodium berghei; Malaria; Merozoite surface; Proteolytic processing; Vaccine

The merozoite surface protein-1 (MSP-1) is a relatively abundant protein which has been found in all Plasmodium species examined [1,2]. MSP-1 is synthesized as a high molecular mass $(\sim 200$ $\mathrm{kDa}$ ) precursor protein which is proteolytically processed into smaller fragments. Proteolytic processing of MSP-1 is a two-step procedure characterized by primary and secondary processing events [3]. Primary processing occurs at, or just before, terminal merozoite differentiation and release and results in the formation of a noncovalent polypeptide complex with fragments of approximately 83, 30, 38 and $42 \mathrm{kDa}$ [4]. The 42

Abbreviations: LT-B, B subunit of E. coli labile toxin; MSP-

1, merozoite surface protein-1; VB1, variable block 1 .

* Corresponding author. Tel.: + 1504 5842507; fax: + 1 504 5996686; e-mail: wiser@mailhost.tcs.tulane.edu

${ }^{1}$ Present address: Department of Biology, City College of San Francisco, San Francisco, CA 94112, USA.

${ }^{2}$ Present address: Michigan Department of Community Health, P.O. Box 30035, Lansing, MI 48909, USA.
$\mathrm{kDa} \mathrm{C}$-terminal fragment is further processed to 33 and $19 \mathrm{kDa}$ fragments at the time of merozoite invasion [5]. This secondary processing is catalyzed by a $\mathrm{Ca}^{2+}$-activated serine protease which results in the shedding of a soluble MSP-1 complex [6]. The remaining glycosyl-phosphatidylinositol-anchored $19 \mathrm{kDa}$ fragment [7] contains two epidermal growth factor-like modules [8] and is carried into the erythrocyte upon merozoite invasion [9].

Comparison of the MSP-1 sequence from $P$. berghei with other rodent parasite MSP-1 sequences [10-12] revealed four major interspecies variable regions, or blocks (Jennings et al., in preparation). The first (i.e., most N-terminal) of these variable blocks was cloned from a $\lambda \mathrm{gt} 11$ cDNA expression library screened with two different mAbs against PbMSP-1 [13]. The mAbs, designated as mAb-F4.4 and mAb-L1.6, were generated from mice immune to $P$. berghei [14]. Variable block 1 (VB1) contains seven degenerate 


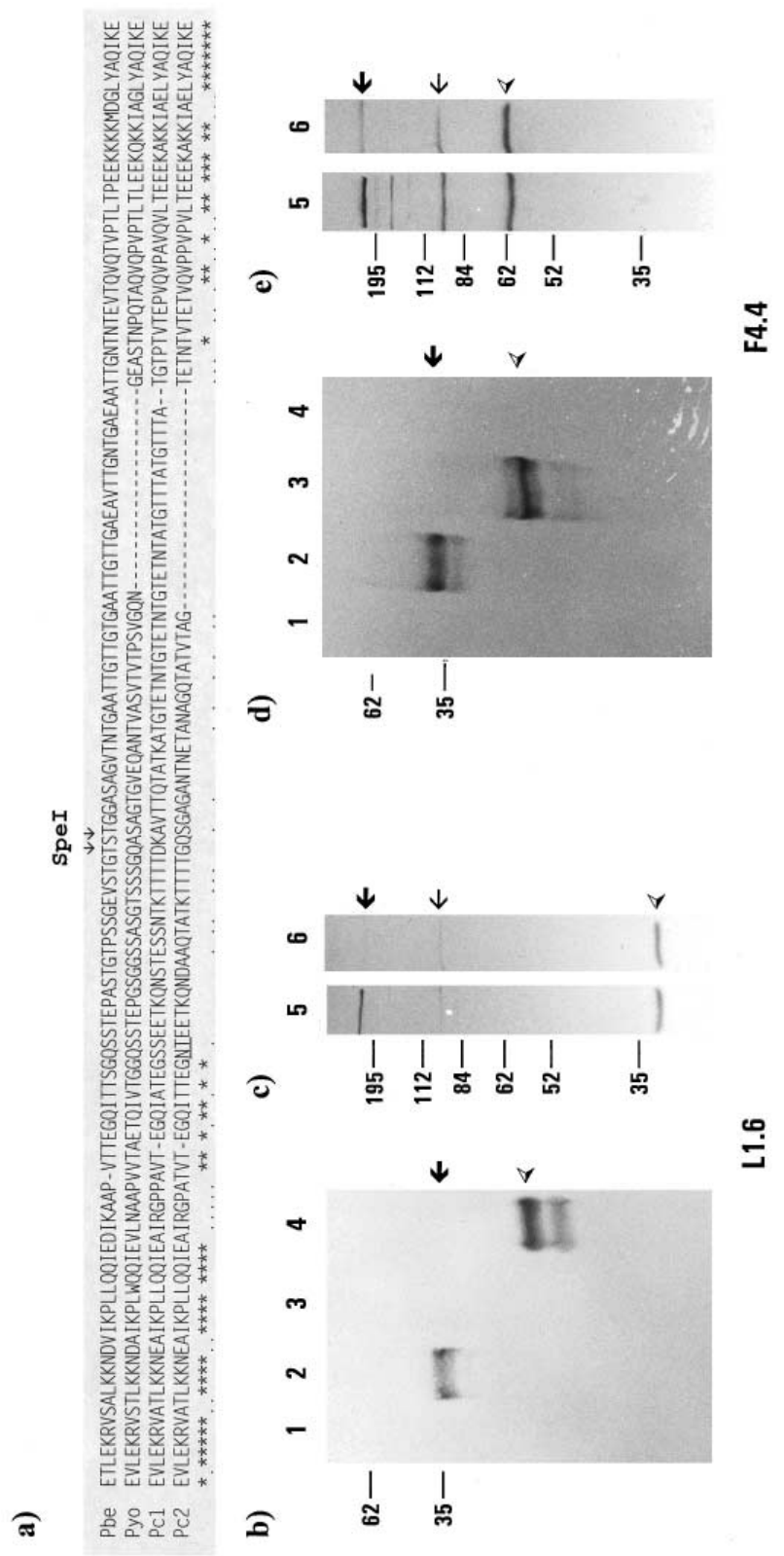

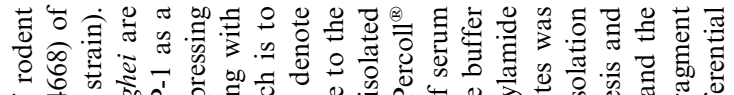

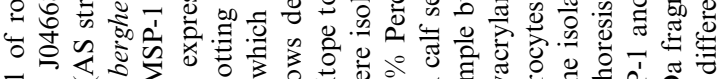



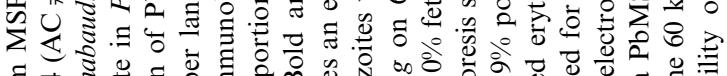

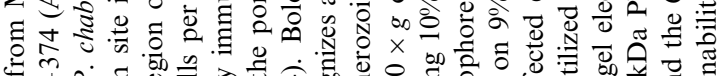
饮

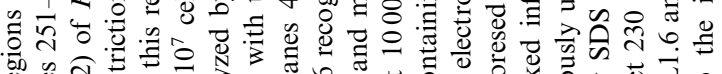

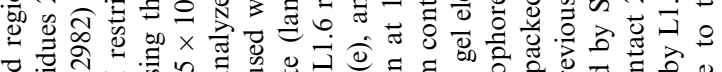

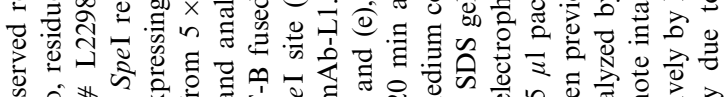



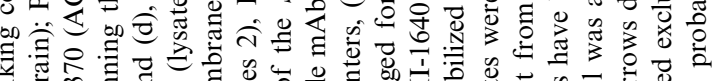

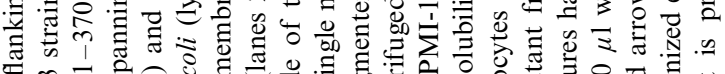



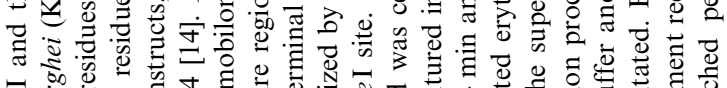



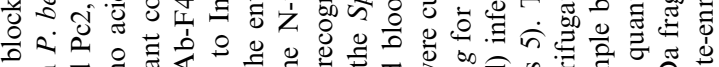

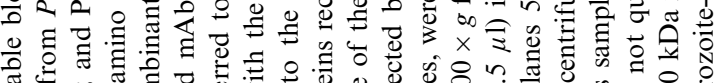

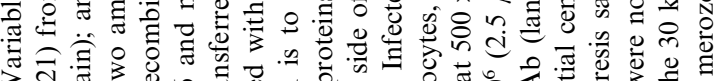

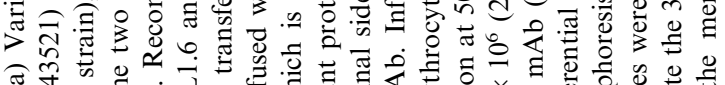

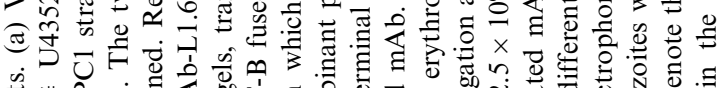

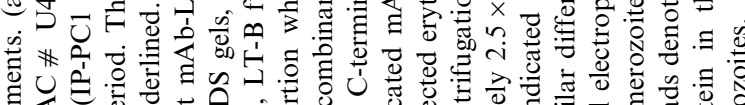

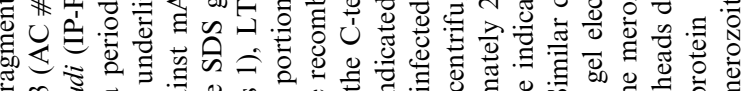

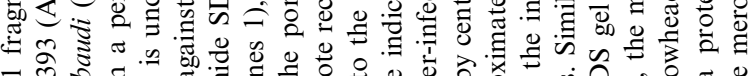

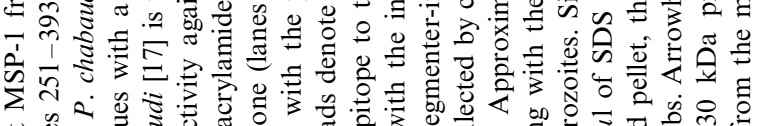



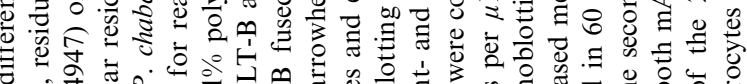

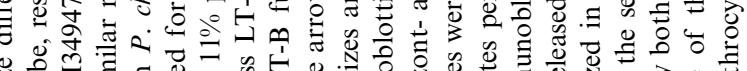

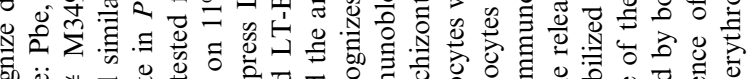
茄

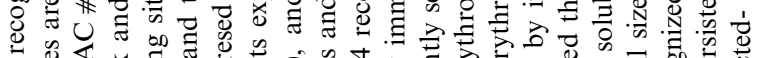



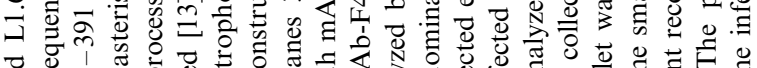
J

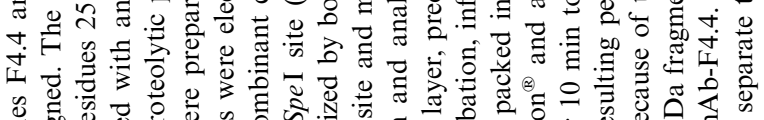

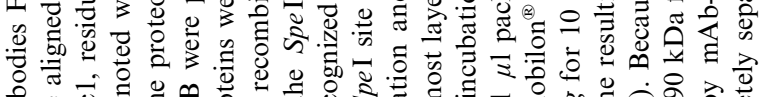

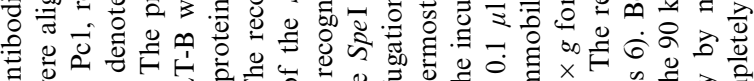

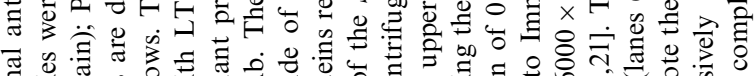

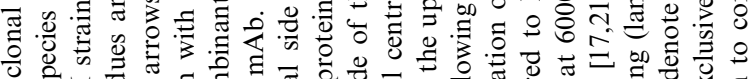



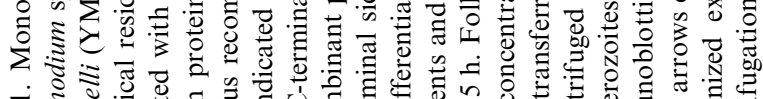
-




tandem repeats of 10 amino acids. A series of recombinant constructs, possessing or lacking the tandem repeats, were prepared by taking advantage of a SpeI restriction site (Fig. 1a), and were tested as vaccines (C.S. Toebe, Ph.D. Dissertation, Tulane University). Immunoblotting of the proteins expressed by these recombinant constructs indicates that the epitope recognized by mAb-L1.6 is to the N-terminal side of the Spe I restriction site (Fig. 1b), and the epitope recognized by mAb-F4.4 is the C-terminal side of the Spe I restriction site (Fig. 1d).

Analysis of schizont-infected erythrocytes and enriched merozoites by SDS gel electrophoresis and immunoblotting reveals that mAb-L1.6 recognizes an approximately $30 \mathrm{kDa}$ polypeptide not recognized by mAb-F4.4 (Fig. 1c), and mAb-F4.4 recognizes an approximately $60 \mathrm{kDa}$ polypeptide not recognized by mAb-L1.6 (Fig. 1e). Both $\mathrm{mAb}-\mathrm{L} 1.6$ and $\mathrm{mAb}-\mathrm{F} 4.4$ recognize the intact 230 kDa MSP-1 and an approximately $90 \mathrm{kDa}$ polypeptide (Fig. 1c,e). This $90 \mathrm{kDa}$ polypeptide is homologous to the $83 \mathrm{kDa}$ processing fragment of P. falciparum MSP-1 as demonstrated by alignment of the rodent Plasmodium MSP-1 sequences with PfMSP-1 sequences [13]. The immunoblotting results indicate that the $90 \mathrm{kDa}$ PbMSP-1 fragment is further processed into $30 \mathrm{kDa}$ and 60 $\mathrm{kDa}$ polypeptides and the protease site is between the L1.6 and F4.4 epitopes. A further processing of the $83 \mathrm{kDa}$ fragment to 73 or $67 \mathrm{kDa}$ fragments has been occasionally reported for PfMSP$1[15,16]$. This further processing of the $83 \mathrm{kDa}$ fragment, however, appears to be a minor event in that the $83 \mathrm{kDa}$ polypeptide is usually the predominant processing product. Therefore MSP-1 from $P$. berghei has a proteolytic processing site that is absent in PfMSP-1.

O'Dea et al. [17] have also proposed an additional protease processing site in the $\mathrm{N}$-terminal region of MSP-1 from $P$. chabaudi which gives rise to 35 and $52 \mathrm{kDa}$ fragments. N-terminal sequencing of the $52 \mathrm{kDa}$ fragment from PcMSP1 reveals that cleavage occurs between an asparagine and a threonine residue (denoted by underlining in Fig. 1a). Alignment of the MSP-1 sequences from other rodent parasites reveals that
Table 1

Distribution of amino acids in the primary processing sites of MSP-1 from rodent Plasmodium species

\begin{tabular}{llllllllll}
\hline P6 & P5 & P4 & P3 & P2 & P1 & P1 $^{\prime}$ & P2' & P3' & P4' \\
\hline $6 \mathrm{I}$ & $4 \mathrm{~V}$ & $6 \mathrm{~A}$ & $4 \mathrm{~S}$ & $10 \mathrm{G}$ & $8 \mathrm{E}$ & $15 \mathrm{~S}$ & $12 \mathrm{E}$ & $6 \mathrm{E}$ & $5 \mathrm{E}$ \\
$4 \mathrm{Q}$ & $4 \mathrm{I}$ & $5 \mathrm{~T}$ & $4 \mathrm{~T}$ & $6 \mathrm{~A}$ & $6 \mathrm{Q}$ & $1 \mathrm{~T}$ & $2 \mathrm{~S}$ & $5 \mathrm{D}$ & $3 \mathrm{~A}$ \\
$3 \mathrm{P}$ & $3 \mathrm{~T}$ & $3 \mathrm{~V}$ & $3 \mathrm{R}$ & & $1 \mathrm{~N}$ & & $2 \mathrm{~T}$ & $2 \mathrm{Q}$ & $3 \mathrm{~T}$ \\
$2 \mathrm{~T}$ & $2 \mathrm{~A}$ & $1 \mathrm{I}$ & $2 \mathrm{E}$ & & $1 \mathrm{~S}$ & & & $2 \mathrm{~T}$ & $2 \mathrm{D}$ \\
$1 \mathrm{H}$ & $1 \mathrm{Q}$ & $1 \mathrm{~S}$ & $2 \mathrm{~N}$ & & & & & $1 \mathrm{~N}$ & $2 \mathrm{~V}$ \\
& $1 \mathrm{R}$ & & $1 \mathrm{G}$ & & & & & & $1 \mathrm{G}$ \\
& $1 \mathrm{~K}$ & & & & & & & &
\end{tabular}

The four rodent Plasmodium sequences were aligned with regard to the four primary processing sites of $P$. chabaudi [17]. Accession numbers for the sequences are listed in the legend to Fig. 1. The number of occurrences of particular amino acids (single-letter code) found at positions surrounding the 16 (four strains $\times$ four primary processing sites) scissile bonds (between $\mathrm{P} 1$ and $\mathrm{P}^{\prime}$ according to the nomenclature of Schechter and Berger [22]) are shown.

the scissile bond is on the boundary between a conserved region and VB1 (Fig. 1a) and that the scissile bonds differ between species and strains. However, the homologous residues in $P$. berghei and $P$. yoelii, glutamine and serine, are chemically similar to asparagine and threonine. $\mathrm{A} \mathrm{mAb}$ against PyMSP-1 immunoprecipitates polypeptides of 230, 90 and $56 \mathrm{kDa}$ and pulse-chase experiments demonstrate that the $230 \mathrm{kDa}$ protein is converted to the 90 and $56 \mathrm{kDa}$ fragments [18]. The similarity in the sizes of the $P$. berghei and $P$. yoelii MSP-1 fragments (i.e. 60 vs. $56 \mathrm{kDa}$ ) suggests that this additional proteolytic processing also occurs in $P$. yoelii. No information is available on the processing of MSP-1 from the IP-PC1 strain of $P$. chabaudi.

The amino acids flanking all four primary processing sites [17] in the MSP-1 sequences from four rodent parasites were analyzed (Table 1). The amino acids surrounding the scissile bonds are somewhat conserved and have a strong predisposition for certain types of amino acids. Polar and/or charged amino acids are found in the P1 and $\mathrm{P} 3$ positions, whereas the $\mathrm{P} 2$ and $\mathrm{P} 4-\mathrm{P} 6$ positions tend to be aliphatic. On the $\mathrm{P}^{\prime}$ side of the scissile bond the amino acids tend to have hydroxyl or acidic side groups giving rise to somewhat negatively charged domain. We propose a 
consensus sequence of $h-h-h-p-G / A-E / Q \downarrow \downarrow S-E-n-$ $\mathrm{n}$, where the three lower case h's refer to a stretch of generally non-polar amino acids in which at least one is hydrophobic, the lower case $\mathrm{p}$ refers to a polar amino acid and the two lower case n's refer to a tendency to be negatively charged. The primary processing sites of PfMSP-1 [17] also exhibit this same general consensus sequence, except that the most $\mathrm{C}$-terminal primary processing sites of PfMSP-1 lacks the strong negative tendency on the $\mathrm{P}^{\prime}$ side of the proposed consensus sequence. However, alternative processing sites for this position, which perfectly match the consensus sequence, have been proposed [19]. In addition, some minor processing fragments have been sequenced [16] and these protease sites also show good, but imperfect, homology to the consensus sequence. Cooper and Bujard [20] have described a schizont membrane associated protease which can cleave recombinant MSP-1. The sequences recognized by this protease all comply with the proposed consensus sequence.

In summary, the collective data indicate that MSP-1 from rodent Plasmodium species has an additional primary processing site that is likely absent in $P$. falciparum. The evidence includes: the similar sizes of the MSP-1 proteolytic fragments $(30-35 \mathrm{kDa}+52-60 \mathrm{kDa})$, the strong amino acid homology in this region (Fig. 1a), and the conservation of the sequence flanking the scissile bond as compared to the other primary processing sites (Table 1). Furthermore, the similarity in the sequences of the primary processing sites suggest that a single protease is responsible for the primary processing. In addition, since they recognize different processing fragments, mAb-L1.6 and mAb-F4.4 will be useful reagents for the study of MSP-1 processing.

\section{Acknowledgements}

We thank Dr John Lonsdale-Eccles (University of Alabama, Birmingham) for helpful discussions on proteases and his critical comments on the manuscript.

\section{References}

[1] Holder, A.A. (1988) The precursor to major merozoite surface antigens: structure and role in immunity. Prog. Allergy 40, 72-97.

[2] Cooper, J.A. (1993) Merozoite surface antigen-1 of Plasmodium. Parasitol. Today 9, 50-54.

[3] Holder, A.A., Blackman, M.J., Burghaus, P.A., Chappel, J.A., Ling, I.T., McCallum-Deighton, N. and Shai, S. (1992) A malaria merozoite surface protein (MSP-1)structure, processing and function. Mem. Inst. Oswaldo. Cruz. 87 (Suppl. III), 37-42.

[4] McBride, J.S. and Heidrich, H.G. (1987) Fragments of the polymorphic $M_{\mathrm{r}} 185000$ glycoprotein form the surface of isolated Plasmodium falciparum merozoites form an antigenic complex. Mol. Biochem. Parasitol. 23, 7184.

[5] Blackman, M.J., Whittle, H. and Holder, A.A. (1991) Processing of the Plasmodium falciparum major merozoite surface protein-1: identification of a 33-kilodalton secondary processing product which is shed prior to erythrocyte invasion. Mol. Biochem. Parasitol. 49, 35-44.

[6] Blackman, M.J. and Holder, A.A. (1992) Secondary processing of the Plasmodium falciparum merozoite surface protein-1 (MSP-1) by a calcium-dependent membranebound serine protease: shedding of $\mathrm{MSP}_{33}$ as a noncovalently associated complex with other fragments of the MSP-1. Mol. Biochem. Parasitol. 50, 307-316.

[7] Gerold, P., Schofield, L., Blackman, M.J., Holder, A.A. and Schwarz, R.T. (1996) Structural analysis of the glycosyl-phosphatidylinositol membrane anchor of the merozoite surface proteins-1 and -2 of Plasmodium falciparum. Mol. Biochem. Parasitol. 75, 131-143.

[8] Blackman, M.J., Ling, I.T., Nichols, S.C. and Holder, A.A. (1991) Proteolytic processing of the Plasmodium falciparum merozoite surface protein-1 produces a membrane bound fragment containing two epidermal growth factor-like domains. Mol. Biochem. Parasitol. 49, 29-34.

[9] Blackman, M.J., Heidrich, H-G, Donachie, S., McBride, J.S. and Holder, A.A. (1990) A single fragment of a malaria merozoite surface protein remains on the parasite during red cell invasion and is the target of invasion inhibiting antibodies. J. Exp. Med. 172, 379-382.

[10] Lewis, A.P. (1990) Sequence analysis of the gene encoding the precursor to the major merozoite surface antigen of Plasmodium yoelii. Mol. Biochem. Parasitol. 39, 285-288.

[11] Deleersnijder, W., Hendrix, D., Bendahman, N., Hanegreefs, J., Hamers-Casterman, C. and Hamers, R. (1990) Molecular cloning and sequence analysis of the gene encoding the major merozoite surface antigen of Plasmodium chabaudi chabaudi IP-PC1. Mol. Biochem. Parasitol. 43, 231-244.

[12] McKean, P.G., O’Dea, K. and Brown, K.N. (1993) Nucleotide sequence analysis and epitope mapping of the merozoite surface protein 1 from Plasmodium chabaudi chabaudi AS. Mol. Biochem. Parasitol. 62, 199-209. 
[13] Toebe, C.S., Clements, J.D., Cardenas, L., Jennings, G.J. and Wiser, M.F. (1997) Recombinant Plasmodium berghei merozoite surface protein-1 expressed in Salmonella: evaluation of immunogenicity in a rodent model. Am. J. Trop. Med. Hyg. 56, in press.

[14] Wiser, M.F. (1986) Characterization of monoclonal antibodies directed against erythrocytic stage antigens of Plasmodium berghei. Eur. J. Cell Biol. 42, 45-51.

[15] Lyon, J.A., Haynes, J.D., Diggs, C.L., Chulay, J.D., Haidaris, C.D. and Pratt-Rossiter, J. (1987) Monoclonal antibody characterization of the $195 \mathrm{kDa}$ major surface glycoprotein of Plasmodium falciparum malaria schizonts and merozoites: identification of additional processed products and a serotype-restricted repetitive epitope. J. Immunol. 138, 895-901.

[16] Stafford, W.H.L., Blackman, M.J., Harris, A., Shai, S., Grainger, M. and Holder, A.A. (1994) N-terminal amino acid sequence of the Plasmodium falciparum merozoite surface protein-1 polypeptides. Mol. Biochem. Parasitol. 66, 157-160.

[17] O'Dea, K.P., McKean, P.G., Harris, A. and Brown, K.N. (1995) Processing of the Plasmodium chabaudi chabaudi
AS merozoite surface protein 1 in vivo and in vitro. Mol. Biochem. Parasitol. 72, 111-119.

[18] Holder, A.A. and Freeman, R.R. (1981) Immunization against blood stage rodent malaria using purified parasite antigens. Nature 294, 361-364.

[19] Cooper, J.A., Cooper, L.T. and Saul, A.J. (1992) Mapping of the region predominantly recognized by antibodies to the Plasmodium-falciparum merozoite surface antigen MSA-1. Mol. Biochem. Parasitol. 51, 301312.

[20] Cooper, J.A. and Bujard, H. (1992) Membrane associated proteases process Plasmodium falciparum merozoite surface antigen-1 (MSA-1) to fragment gp41. Mol. Biochem. Parasitol. 56, 151-160.

[21] Braun-Breton, C., Blisnick, T., Jouin, H., Barale, J.C., Rabilloud, T., Langsley, G. and Da Silva, L.H.P. (1992) Plasmodium chabaudi p68 serine protease activity required for merozoite entry into mouse erythrocytes. Proc. Natl. Acad. Sci. USA 89, 9647-9651.

[22] Schechter, I. and Berger, A. (1967) On the active site in proteases. I. Papain. Biochem. Biophys. Res. Commun. $27,157-162$. 